\title{
The Role of Limited Respiration in the Incomplete Oxidation of Glucose by Saccharomyces cerevisiae
}

\author{
By MARTIN RIEGER, OTHMAR KÄPPELI* AND ARMIN FIECHTER \\ Department of Biotechnology, Swiss Federal Institute of Technology, Hönggerberg, \\ CH-8093 Zürich, Switzerland
}

(Received 18 May 1982; revised 2 August 1982)

\begin{abstract}
The respiratory capacity of Saccharomyces cerevisiae growing in continuous culture on glucose and on mixtures of glucose and ethanol was investigated. An oxygen uptake rate of $8 \mathrm{mmol}$ $\mathrm{g}^{-1} \mathrm{~h}^{-1}$ was found to limit the ability of the organism to degrade a substrate purely oxidatively. On glucose as sole energy and carbon source, this respiration rate was invariably achieved at an identical growth rate and thus at an identical substrate uptake rate when the inlet glucose concentration was varied. The rate of ethanol co-consumption together with glucose was strictly governed by this limiting maximum respiratory capacity and no repression of respiration was observed at dilution rates where ethanol was excreted by the cells. Hence, a limitation in some step in the oxidative branch of catabolism is likely to be responsible for incomplete oxidation of glucose at high growth rates rather than an undefined action of glucose repression.
\end{abstract}

\section{INTRODUCTION}

When Saccharomyces cerevisiae is grown in a batch culture under aerobic conditions with glucose as sole energy and carbon source, ethanol is always released into the culture liquid as a by-product. The ethanol can subsequently be assimilated in a second growth phase after glucose is completely used and the cells have adapted to the new carbon source. However, when the same organism is cultivated in a chemostat, a strictly oxidative metabolism can be observed at low dilution rates. This results in exclusive formation of biomass and carbon dioxide in significant quantities. When a particular critical dilution rate is surpassed in the chemostat, the cells are no longer able to degrade all the substrate consumed oxidatively. As a consequence, ethanol appears in the culture liquid and the metabolism becomes predominantly glycolytic, the amount of biomass produced per unit of substrate consumed decreases steadily and the respiratory quotient increases with increasing growth rate (von Meyenburg, 1969; Fiechter et al., 1981).

This switch in the overall metabolic state at high glucose concentrations has been designated as the Crabtree effect, or the glucose effect (Crabtree, 1929; De Deken, 1966; Polakis \& Bartley, 1965). It is accompanied by a variety of individual biochemical control processes, including the repression of several tricarboxylic acid cycle enzymes, alterations in the content of components of the electron transport unain, repression of the enzymes of the gluconeogenetic pathway, and even a proteolytic inactivation of some repressible (iso-) enzymes (Polakis \& Bartley, 1965; Polakis et al., 1965; Witt et al., 1966; Beck \& von Meyenburg, 1968; Haarasilta \& Oura, 1975; Holzer, 1976; Fiechter et al., 1981).

The investigation of yeast strains carrying different mutations has shown that some of the regulatory events of the glucose effect may be absent in one strain while present in another, although both strains exhibit normal sensitivity to glucose regarding the repression of respiration and the concomittant release of ethanol (Entian, 1977; Ciriacy \& Breitenbach, 1979). Consequently, no mechanism on a molecular level which would account for the variety of regulatory events concerned with the glucose effect has emerged so far. 
The fact that fully derepressed growth of $S$. cerevisiae on glucose can also be observed in a chemostat culture indicates that the primary cause of repression is not the presence of the substrate per se, but rather the metabolic flux generated by the uptake of substrate into the cell (Schatzmann, 1975; Bijkerk \& Hall, 1977; Barford \& Hall, 1979b). This would indicate that there must be a particular substrate uptake rate for a certain strain of $S$. cerevisiae which would be characteristic of the onset of the glucose effect in this organism. The results taken from the available literature fail to demonstrate such a characteristic value (Karrer, 1978).

We investigated this problem by cultivating a strain of baker's yeast under constant conditions except for varying the inlet glucose concentration. In addition, a possible limited respiratory capacity under non-repressive growth conditions was investigated by determining the rate of ethanol co-consumption when growing this organism on mixtures of glucose and ethanol.

\section{METHODS}

Organism and growth conditions. The yeast strain Saccharomyces cerevisiae H1022 (Department of Biotechnology, ETH Zürich) was grown with glucose and with mixtures of glucose and ethanol as carbon source. The synthetic medium contained $\left(\mathrm{mol}\right.$ carbon $\left.{ }^{-1}\right):\left(\mathrm{NH}_{4}\right)_{2} \mathrm{SO}_{4}, 6 \mathrm{~g} ;\left(\mathrm{NH}_{4}\right)_{2} \mathrm{HPO}_{4}, 1.95 \mathrm{~g} ; \mathrm{KCl}, 0.9 \mathrm{~g} ; \mathrm{MgSO}_{4} .7 \mathrm{H}_{2} \mathrm{O}$, $0.45 \mathrm{~g} ; \mathrm{CaCl}_{2} .2 \mathrm{H}_{2} \mathrm{O}, 0.3 \mathrm{~g} ; \mathrm{CuSO}_{4} .5 \mathrm{H}_{2} \mathrm{O}, 2.34 \mathrm{mg} ; \mathrm{FeCl}_{3} .6 \mathrm{H}_{2} \mathrm{O}, 14.4 \mathrm{mg} ; \mathrm{ZnSO}_{4} .7 \mathrm{H}_{2} \mathrm{O}, 9 \mathrm{mg} ; \mathrm{MnSO}_{4} .2 \mathrm{H}_{2} \mathrm{O}$, $10.5 \mathrm{mg}$; biotin, $0.03 \mathrm{mg}$; myo-inositol, $60 \mathrm{mg}$; calcium pantothenate, $30 \mathrm{mg}$; thiamin hydrochloride, $6 \mathrm{mg}$; pyridoxine hydrochloride, $1.5 \mathrm{mg}$; and $0.25 \mathrm{ml}$ of polypropylene glycol 2000 (Fluka, Switzerland) was added as an antifoam agent. Medium NL18 was prepared according to von Meyenburg (1969). A glass reactor with 2.51 working volume was equipped with automatic $\mathrm{pH}$ and temperature control. The $\mathrm{pH}$ was kept constant at 5.0 by addition of $5 \mathrm{M}-\mathrm{KOH}$. The cultivation temperature was $30^{\circ} \mathrm{C}$.

Product analysis. Dry cell weight was determined by removing $10 \mathrm{ml}$ samples from the bulk of the culture liquid. The cells were collected by centrifugation at $2000 \mathrm{~g}$ for $5 \mathrm{~min}$ in previously tared centrifuge tubes. The sediment was washed twice with distilled water and then dried to constant weight at $105^{\circ} \mathrm{C}$.

Glucose was determined enzymically by a commercially available test kit (GOD-Perid test, Boehringer, Mannheim, F.R.G.). Ethanol was analysed with a gas chromatograph (Hewlett Packard, model 5830A, Avondale, Pa., U.S.A.). Prior to injection, the cells were removed by centrifugation and a known amount of methanol was added as internal standard. The column was Propack QS, mesh 100-120, stainless steel $6 \mathrm{ft}(1.8 \mathrm{~m})$. The temperature was $150^{\circ} \mathrm{C}$.

The elemental composition of the cells was determined by the Laboratory of Organic Chemistry (Swiss Federal Institute of Technology, Zürich). The composition of biomass was found to be virtually constant in the dilution rate range used in this study. All stoichiometric calculations were therefore based on the following formula: $\mathrm{C}_{1} \mathrm{H}_{1 \cdot 79} \mathrm{O}_{0.57} \mathrm{~N}_{0.15}$

Calculation of the stoichiometric coefficients and gas turnover rates. Growth on glucose, which results in the formation of significant amounts of only biomass, ethanol, carbon dioxide and water, can be described as (Herbert, 1975):

$$
\mathrm{C}_{6} \mathrm{H}_{12} \mathrm{O}_{6}+\alpha_{1} \mathrm{O}_{2}+0 \cdot 15 \alpha_{2} \mathrm{NH}_{3} \rightarrow \alpha_{2} \mathrm{C}_{1} \mathrm{H}_{1 \cdot 79} \mathrm{O}_{0 \cdot 57} \mathrm{~N}_{0 \cdot 15}+\alpha_{3} \mathrm{C}_{2} \mathrm{H}_{6} \mathrm{O}+\alpha_{4} \mathrm{CO}_{2}+\alpha_{5} \mathrm{H}_{2} \mathrm{O}
$$

and the corresponding yield quotients [expressed as (mol product-C formed)/(mol substrate-C used)], become:

$$
Y_{\mathrm{S}}^{\mathbf{X}}=\alpha_{2} / 6 \quad Y_{\mathrm{S}}^{\mathrm{Co}_{2}}=\alpha_{4} / 6 \quad Y_{\mathrm{S}}^{\mathrm{Et}}=\alpha_{3} / 3 \quad Y_{\mathrm{O}_{2}}^{\mathrm{X}}=\alpha_{2} / \alpha_{1}
$$

hence

$$
\alpha_{2}=6 Y_{\mathrm{s}}^{\mathrm{x}} \quad \alpha_{4}=6 Y_{\mathrm{s}}^{\mathrm{co}_{2}} \quad \alpha_{3}=3 Y_{\mathrm{s}}^{\mathrm{Et}}
$$

$Y_{\mathrm{S}}^{\mathrm{X}}$ and $Y_{\mathrm{S}}^{\mathrm{Et}}$ can be obtained from the steady state concentrations for substrate, biomass and ethanol. $Y_{\mathrm{S}}^{\mathrm{CO}_{2}}$ is derived from the carbon balance equation:

$$
Y_{\mathbf{s}}^{\mathrm{x}}+Y_{\mathrm{s}}^{\mathrm{Co}_{2}}+Y_{\mathbf{s}}^{\mathrm{Et}}=1
$$

$\alpha_{1}$ and $\alpha_{5}$ are calculated from the mass balance equations for oxygen and hydrogen respectively:

$$
\begin{gathered}
\text { Hydrogen: } \begin{array}{r}
12+0.45 \alpha_{2}=1.79 \alpha_{2}+6 \alpha_{3}+2 \alpha_{5} \\
\alpha_{5}=6-0.67 \alpha_{2}-3 \alpha_{3}
\end{array} \\
\text { Oxygen: } \quad 3+\alpha_{1}=0.5\left(0.57 \alpha_{2}+\alpha_{3}+2 \alpha_{4}+\alpha_{5}-6\right) \\
\alpha_{1}=0.5\left(0.57 \alpha_{2}+\alpha_{3}+2 \alpha_{4}+\alpha_{5}-6\right)
\end{gathered}
$$


The specific gas turnover rates were obtained as follows:

$$
\begin{array}{lcl}
\mathrm{d} x / \mathrm{d} t=\mu x & \mathrm{~d} x / \mathrm{d} s=\mu / q_{\mathrm{s}}=Y_{\mathrm{s}}^{\mathrm{x}} & \\
\mathrm{d} s / \mathrm{d} t=q_{\mathrm{s}} x & \\
\mathrm{dCO} / \mathrm{d} t=q_{\mathrm{CO}_{2}} x & \mathrm{dCO}_{2} / \mathrm{d} s=q_{\mathrm{Co}_{2}} / q_{\mathrm{s}}=Y_{\mathrm{s}}^{\mathrm{CO}_{2}} & q_{\mathrm{Co}_{2}}=D\left(Y_{\mathrm{s}}^{\mathrm{Co}_{2} / Y_{\mathrm{s}} \mathrm{x}}\right) \\
\mathrm{dO}_{2} / \mathrm{d} t=q_{\mathrm{O}_{2}} x & \mathrm{~d} x / \mathrm{dO}_{2}=\mu / q_{\mathrm{O}_{2}}=Y_{\mathrm{O}_{2}}^{\mathrm{x}} & q_{\mathrm{O}_{2}}=D\left(1 / Y_{\mathrm{O}_{2}}^{\mathrm{x}}\right)
\end{array}
$$

and

$$
\begin{aligned}
Q_{\mathrm{O}_{2}} & =q_{\mathrm{O}_{2}}[(1000 \times 0.47) / 12] \\
Q_{\mathrm{Co}_{2}} & =q_{\mathrm{Co}_{2}}[(1000 \times 0.47) / 12] \\
|Q| & =\mathrm{mmol} \mathrm{g}^{-1} \mathrm{~h}^{-1}
\end{aligned}
$$

\section{RESULTS}

\section{Growth at three different inlet glucose concentrations}

The chemostat cultures of $S$. cerevisiae $\mathrm{H} 1022$ grown at the three different inlet glucose concentrations of 5,10 , and $30 \mathrm{~g} \mathrm{l}^{-1}$ showed good correspondence of biomass versus dilution rate profiles (Fig. 1 $a$ ). In all three cases the metabolism was completely oxidative up to a dilution rate of $D_{\mathbf{R}}=0.3 \pm 0.005 \mathrm{~h}^{-1}$ and the growth yield in this range was constant at $0.48 \pm 0.005$. A glucose uptake rate of $3.45 \pm 0.005 \mathrm{mmol} \mathrm{g}^{-1} \mathrm{~h}^{-1}$ (Fig. 1c) and an oxygen uptake rate of $8.1 \pm$ $0.2 \mathrm{mmol} \mathrm{g}^{-1} \mathrm{~h}^{-1}$ were found to be characteristic of the onset of repression in this organism (Fig. 1 b). No decrease of respiratory activity could be detected at dilution rates higher than $0 \cdot 3 \mathrm{~h}^{-1}$. The maximum value reached at $D_{\mathrm{R}}$ was maintained up to a dilution rate of $0.41 \mathrm{~h}^{-1}$ in all three cultures. This is in contrast to earlier findings which reported a strong decrease of respiratory activity in this organism at high growth rates (von Meyenburg, 1969). The reason for this deviation from previous results is not clear so far. However, comparable results were obtained from the simultaneous determination of oxygen uptake rates by analysis of the exhaust air in a gas analyser. The carbon dioxide production rates corresponded well to those reported by von Meyenburg (1969). Due to the higher oxygen uptake rates observed at glucose-repressed growth, the respiratory quotient did not exceed the value of 4 , which is 3 to 4 times smaller than the respiratory quotients reported by von Meyenburg (1969).

The specific gas turnover rates and the growth yield of the cultures with 5,10 , and $30 \mathrm{~g} \mathrm{l}^{-1}$ glucose displayed a similar response in function of the dilution rate (Table 1 ). Thus the curves for $Q_{\mathrm{S}}$ and $Q_{\mathrm{O}_{2}}$ coincided, indicating an identical overall metabolic state of the organism at a particular growth rate, irrespective of the inlet glucose concentration (Fig. 1b). In the range of glucose-derepressed growth, the equilibrium glucose concentration was close to the detection limit, independent of the inlet glucose concentration (Table 1). Due to this analytical difficulty, no clear correlation between the dilution rate and the residual glucose concentration was obvious in this range. Under glucose-repressed conditions, a tendency for increased residual substrate concentrations was observed with the higher inlet glucose concentrations. In this region, an increased scattering of the steady state values for biomass and glucose concentrations during cultivation periods of several days was characteristic (Table 1). In contrast, the biomass yields on glucose were rather constant and deviations were always in the range of $5 \%$. As a consequence, the values for the metabolic turnover rates (specific substrate and oxygen uptake and carbon dioxide production rates) were remarkably similar.

\section{Growth on mixtures of glucose and ethanol}

In order to determine the respiratory capacity of $S$. cerevisiae, the cells were grown on mixtures of glucose and ethanol. Since ethanol is degraded oxidatively only, the growth on substrate mixtures should indicate the role of the respiratory activity in the breakdown of glucose. For the experiments, the two carbon sources were used at different concentrations : mixture A contained $\left(\mathrm{l}^{-1}\right) 15 \mathrm{~g}$ ethanol and $15 \mathrm{~g}$ glucose; mixture $\mathrm{B}$ contained $\left(\mathrm{l}^{-1}\right) 5 \mathrm{~g}$ ethanol and $15 \mathrm{~g}$ glucose. The 


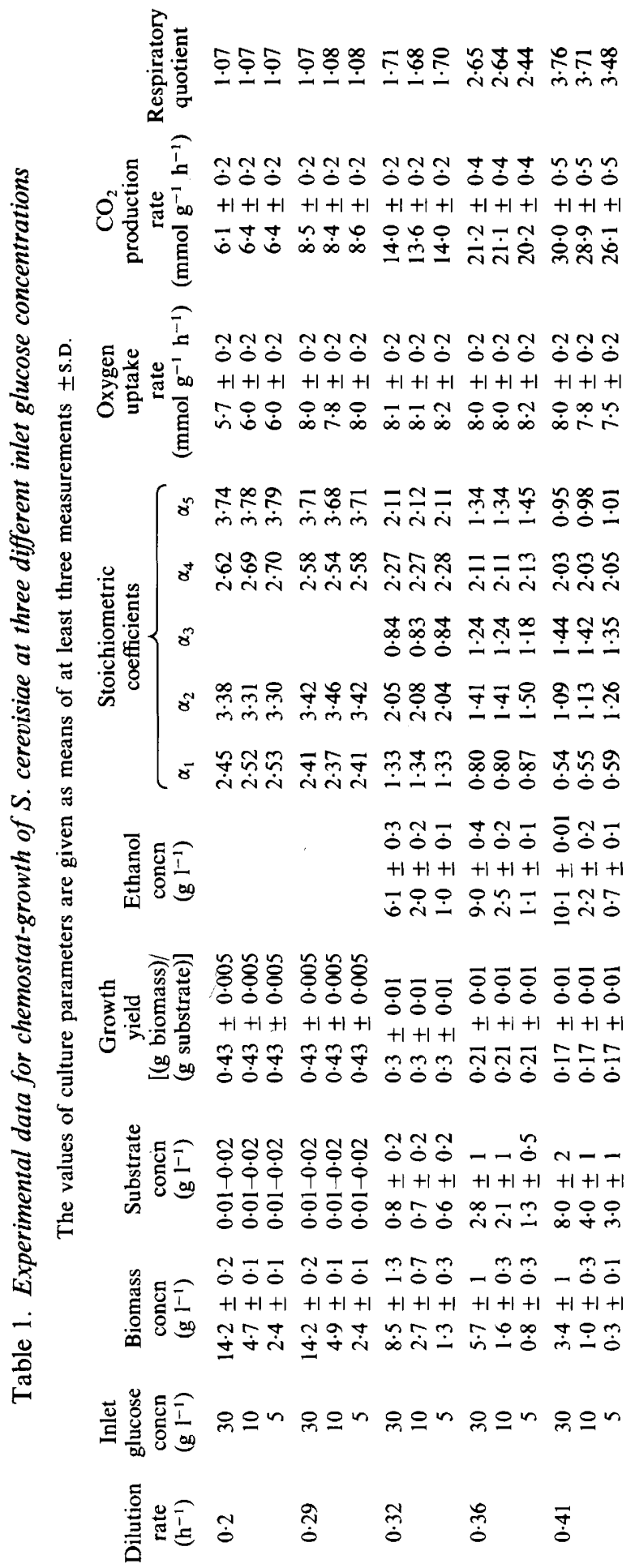




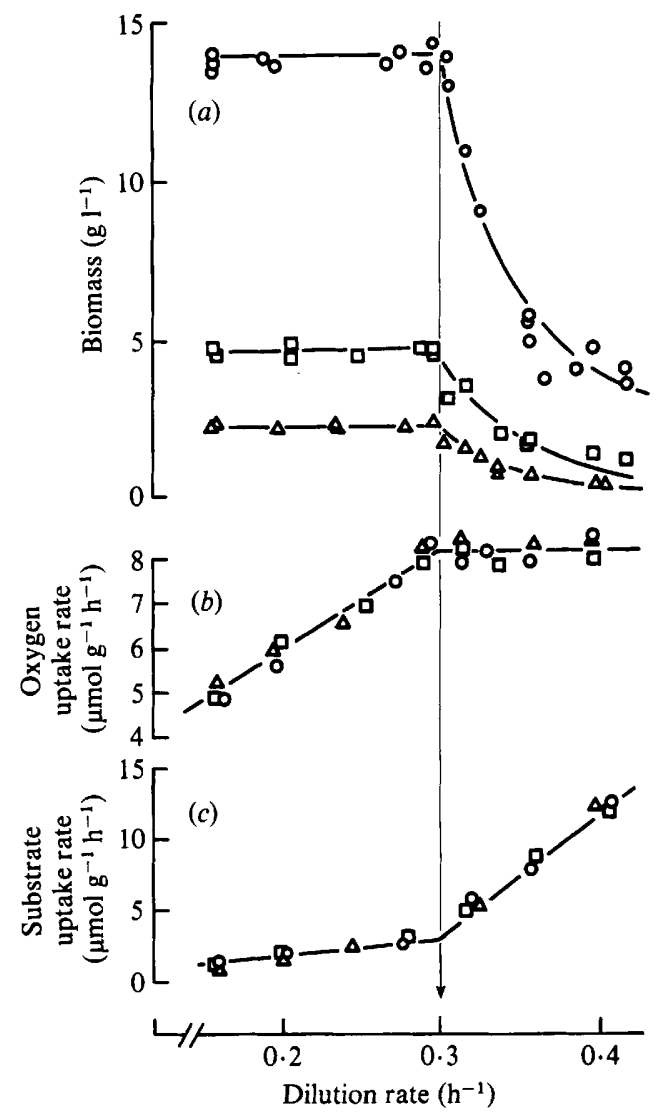

Fig. 1. Continuous culture of $S$. cerevisiae with glucose as the limiting substrate. (a) Biomass, $(b)$ oxygen uptake rate and $(c)$ substrate uptake rate. The inlet glucose concentrations were $30 \mathrm{~g} \mathrm{l}^{-1}(\mathrm{O}), 10 \mathrm{~g} \mathrm{l}^{-1}$ ( $\square)$ and $5 \mathrm{~g}^{-1}(\triangle)$. The arrow indicates the onset of ethanol excretion.

formation of biomass as a function of dilution rate is shown in Fig. 2. For both mixtures, maximum growth yield was observed up to a particular dilution rate designated as $D_{\mathrm{A}}$ and $D_{\mathrm{B}}$ for mixtures A and B respectively. $D_{\mathrm{A}}$ was determined ( \pm S.D.) as $0.23 \pm 0.005 \mathrm{~h}^{-1}$ and $D_{\mathrm{B}}$ as $0.27 \pm$ $0.005 \mathrm{~h}^{-1}$. They were the highest growth rates in which no ethanol was detectable in the culture broth (Fig. 3). The oxygen uptake rates of the cultures increased steadily to the maximum value of $8.0 \pm 0.2 \mathrm{mmol} \mathrm{g}^{-1} \mathrm{~h}^{-1}$, which they reached at $D_{\mathrm{A}}$ and $D_{\mathrm{B}}$, respectively. This is the same maximum $Q_{\mathrm{O}_{2}}$ which was found in glucose-grown cultures, but it was attained at remarkably lower growth rates. As on glucose, no further enhancement of oxygen uptake was observed when the dilution rate was increased further. When the dilution rate was increased above $D_{\mathrm{A}}$ or $D_{\mathrm{B}}$ in the respective cultures, ethanol occurred in the culture liquid. At a dilution rate of $0.3 \mathrm{~h}^{-1}$, the ethanol concentration of the culture liquid was equal to the inlet ethanol concentration, indicating that ethanol was neither consumed nor produced by the cells at this state (Fig. 3). The ethanol uptake rates reached their maximum at $D_{\mathrm{A}}$ and $D_{\mathrm{B}}$ in the respective cultures and then dropped steadily with increasing growth rates (Fig. 4). At dilution rates above $0 \cdot 3 \mathrm{~h}^{-1}$, ethanol was released from the cells in the same way as it was released in cultures where glucose was the only carbon source.

The growth yields in the range of complete substrate oxidation were $0.62-0.63$ on mixture A and $0.6-0.61$ on mixture $B$. They were intermediate values of the characteristic growth yields of this organism on each single substrate. The growth yield on ethanol was determined as 0.72 in a chemostat culture (results not shown). 


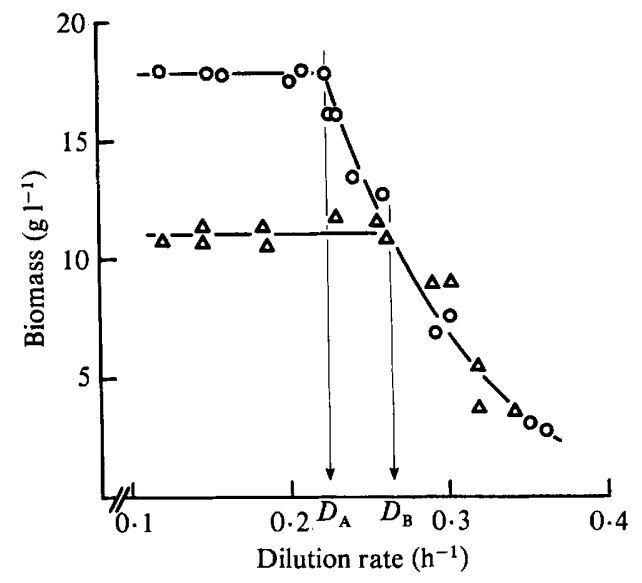

Fig. 2

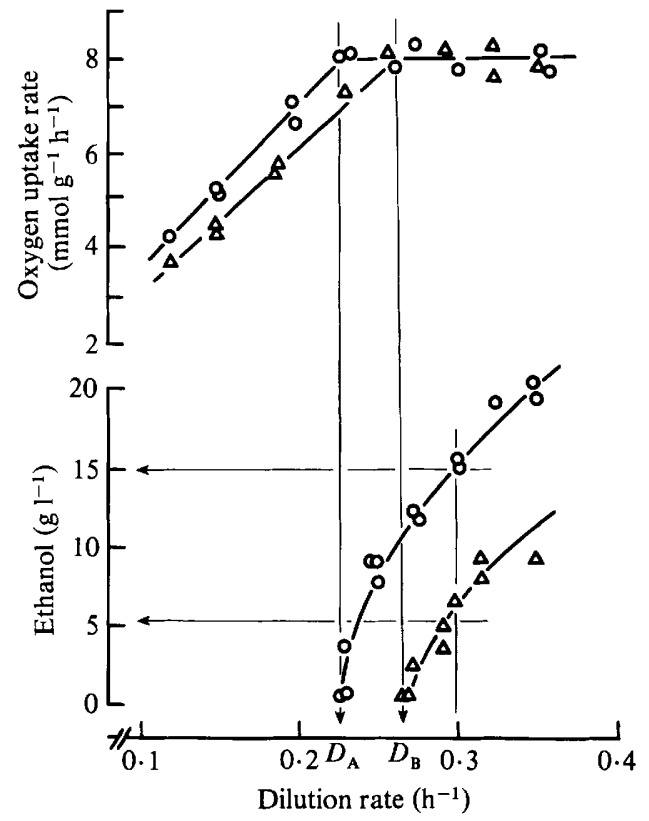

Fig. 3

Fig. 2. Continuous culture of $S$. cerevisiae with mixtures of glucose and ethanol as substrates. $O$, Mixture $A\left(1^{-1}\right): 15 \mathrm{~g}$ glucose $+15 \mathrm{~g}$ ethanol; $\triangle$, mixture $\mathrm{B}\left(\mathrm{l}^{-1}\right): 15 \mathrm{~g}$ glucose $+5 \mathrm{~g}$ ethanol. Arrows indicate the maximum growth rate up to which complete consumption of both carbon sources was observed in the respective culture.

Fig. 3. Specific oxygen uptake rates and ethanol contents of the culture liquid for the growth of $S$. cerevisiae on the two glucose/ethanol mixtures. $O$, Mixture A $\left(1^{-1}\right): 15 \mathrm{~g}$ glucose $+15 \mathrm{~g}$ ethanol; $\triangle$, mixture $\mathrm{B}\left(1^{-4}\right): 15 \mathrm{~g}$ glucose $+5 \mathrm{~g}$ ethanol.

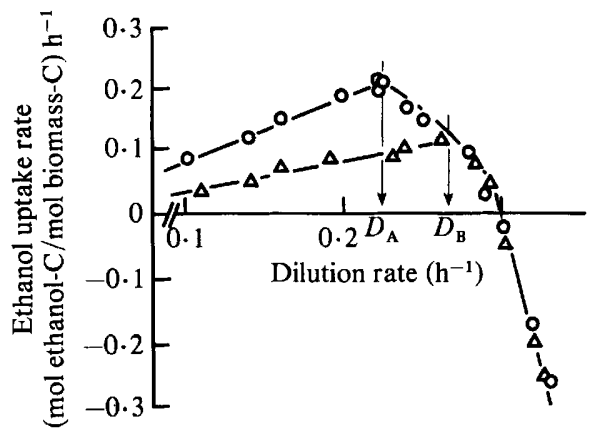

Fig. 4. Ethanol consumption and production rates for growth of $S$. cerevisiae on the two glucose/ethanol mixtures. $\bigcirc$, Mixture A $\left(1^{-1}\right): 15 \mathrm{~g}$ glucose $+15 \mathrm{~g}$ ethanol; $\triangle$, mixture $\mathrm{B}\left(1^{-1}\right): 15 \mathrm{~g}$ glucose $+5 \mathrm{~g}$ ethanol.

\section{DISCUSSION}

Various data have been published on the change from completely oxidative metabolism to incomplete oxidation of glucose in continuous cultures of $S$. cerevisiae $\mathrm{H} 1022$. The critical dilution rate $D_{\mathrm{R}}$, when ethanol excretion begins, has been reported as $0.25 \mathrm{~h}^{-1}$ (von Meyenburg, 1969), $0 \cdot 3 \mathrm{~h}^{-1}$ (Knoepfel, 1972), $0 \cdot 24 \mathrm{~h}^{-1}$ (Karrer, 1978), and even as being dependent on inlet 


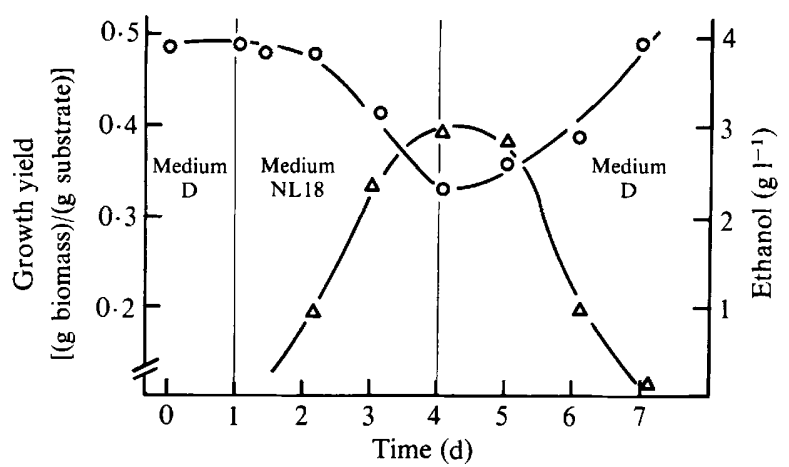

Fig. 5. Growth of $S$. cerevisiae on two different media, medium D and medium NL18. O, Growth yield; $\triangle$, ethanol concentration.

glucose concentration (Karrer, 1978). We found that in order to reach the highest respiratory growth rate of $0.3 \mathrm{~h}^{-1}$ on the $3 \%$ glucose medium, it was necessary to keep dilution rate shifts increasingly small when approaching $D_{\mathrm{R}}$. When steps larger than $0.01 \mathrm{~h}^{-1}$ were applied in a dilution rate range close to $D_{\mathrm{R}}$, repression was frequently triggered, as indicated by the appearance of ethanol in the culture broth. After a short phase of ethanol production, the culture was able to fully re-establish oxidative growth only at dilution rates below $0.25 \mathrm{~h}^{-1}$. In the dilution rate range between $0.25 \mathrm{~h}^{-1}$ and $0.3 \mathrm{~h}^{-1}$, this return to complete oxidative growth was not observed within 2 to $3 \mathrm{~d}$. Although the effect was not fully quantified, we assume that it was due to a short-term increase of the actual glucose concentration in the culture liquid at the time the medium flux rate was enhanced. This effect was most distinct in the $3 \%$ medium and virtually absent in the $0.5 \%$ medium, and led to an erroneous identification of $D_{\mathrm{R}}$ in the case of the $3 \%$ medium at the beginning of our work. Moreover, this technical peculiarity might be responsible for the reported dependence of $D_{\mathrm{R}}$ on the inlet glucose concentration (Karrer, 1978). When this sensitivity towards dilution rate shifts was properly taken into account, a similar overall situation of the metabolism was observed, depending only on the substrate supply rate but not related to the inlet glucose concentration. This was clearly evident from the similarity of the stoichiometric coefficients of the three cultures (Table 1).

In contrast to earlier investigations which usually reported a strong decrease of respiratory activity at high glucose feed rates, we were never able to demonstrate such a regulatory effect on respiration in this organism (Beck \& von Meyenburg, 1968; De Deken, 1966; von Meyenburg, 1969 ; Knoepfel, 1972). We only found a limitation of the maximum oxygen uptake rate at about $8 \mathrm{mmol} \mathrm{g}^{-1} \mathrm{~h}^{-1}$. We therefore checked the growth of $S$. cerevisiae $\mathrm{H} 1022$ on the medium NL18 which was employed by von Meyenburg (1969). This medium cannot be designated as fully synthetic since it requires the addition of $0.75 \mathrm{~g}$ yeast extract per $30 \mathrm{~g}$ glucose to enable growth of the yeast. We found that, if this complex supplement was omitted, virtually no growth was detectable in this medium. Upon addition of $10 \mathrm{mg} \mathrm{MnSO} \mathrm{M}_{4} \cdot 1 \mathrm{H}_{2} \mathrm{Ol}^{-1}$, the organism returned to normal growth kinetics, indicating that manganese might have been limiting in the original NL18. A shift experiment at $D=0.28 \mathrm{~h}^{-1}$ from medium D to NL18 and back elucidated the decisive role of the medium composition in relation to growth kinetics (Fig. 5). Within $3 \mathrm{~d}$ growth yield and $Q_{\mathrm{O}_{2}}$ dropped about $30 \%$ and ethanol was formed. This repression was reversed when NL18 was replaced by medium D. This is a strong hint of a possible limitation, most probably of manganese, in the original NL18 and raises doubts about the significance of the previous results. The reliability of the stoichiometric description of the growth process (Herbert, 1975) was assessed by calculating the carbon balance in the $3 \%$ medium. The recovery was $97-100 \%$ and better than $94 \%$ for respiratory and reductive growth, respectively. The estimations of specific gas turnover rates were consequently based on these values, since they showed much smaller deviations and better reproducibility than those derived from gas analyser readings. This is due to the fact that the differences in oxygen content between inlet and outlet airstream became very small, particularly at low dilution rates and biomass concentrations, thus 
amplifying the effect of random and systematic errors in calculations of the respective turnover rates (Barford \& Hall, 1979a).

In a batch culture, the cells can consume ethanol only after the medium has been completely depleted of glucose. In contrast, in a continuous culture the organism is able to oxidize both substrates simultaneously under conditions where the respective glucose uptake rate does not exceed $3.5 \mathrm{mmol} \mathrm{g}^{-1} \mathrm{~h}^{-1}$ (Geurts et al., 1980). These conditions are attainable in a chemostat culture at dilution rates below $0 \cdot 3 \mathrm{~h}^{-1}$ where the glucose concentrations are low (at about 10$20 \mathrm{mg} \mathrm{l}^{-1}$ ). Comparable conditions are never achieved in a batch culture where high substrate concentrations always cause high uptake rates and, consequently, the formation of ethanol, analogous to a chemostat culture at high dilution rates (Fiechter et al., 1981).

While glucose is always the preferred substrate, the co-consumption of ethanol is clearly governed by the maximum respiration rate attainable by this organism. Once maximal respiration is established, additional ethanol is rejected by the cells. This observation strongly suggests that a limitation of the respiratory capacity might be an inherent feature of the organism and could be independent of the presence of glucose as a substrate. This is supported by the observation that no decrease in oxygen uptake rate was detected in the reductive growth range when the substrate was glucose or carbon source mixtures. Under all conditions investigated, once the maximum respiration rate was reached, it was maintained when the substrate supply rate was increased further. A similar observation was reported by Barford \& Hall (1979b). They found an identical maximum respiration rate on glucose and on galactose and also were not able to detect any repressive effect of high glucose consumption rates on the respiratory activity of their strain of baker's yeast.

These observations offer a new insight into the regulatory phenomenon of glucose metabolism known as the glucose effect. It indicates that a simple limitation in a step of the oxidative pathways leads to a switch in the overall catabolism of glucose and is not some obscure, glucoserelated mechanism.

We have initiated the necessary kinetic work in order to clarify the events accompanying the transition from oxidative breakdown to incomplete oxidation of glucose in this yeast.

This research was supported by grants of the Swiss National Foundation, nos 3.465-079 and 3.051-081.

\section{REFERENCES}

BARFORD, J. P. \& HALL, R. J. (1979a). Investigation of the significance of a carbon and redox balance to the measurement of gaseous metabolism in Saccharomyces cerevisiae. Biotechnology and Bioengineering 21, 609-626.

BARFORD, J. P. \& HALl, R. J. (1979b). An examination of the Crabtree Effect in Saccharomyces cerevisiae: the role of respiratory adaptation. Journal of General Microbiology 114, 267-275.

BeCK, C. \& Von Meyenburg, H. K. (1968). Enzyme pattern and aerobic growth of Saccharomyces cerevisiae under various degrees of glucose limitation. Journal of Bacteriology 96, 479-486.

BiJkerK, A. H. E. \& HALL, R. J. (1977). A mechanistic model for the aerobic growth of Saccharomyces cerevisiae. Biotechnology and Bioengineering 19, 267-296.

Ciriacy, M. \& Breitenbach, I. (1979). Physiological effects of seven different blocks in glycolysis in Saccharomyces cerevisiae. Journal of Bacteriology 139 , 152-160.

Crabtree, H. G. (1929). Observations on the carbohydrate metabolism of tumors. Biochemical Journal 23, 536-545.
De Deken, R. H. (1966). The Crabtree Effect. A regulatory system in yeast. Journal of General Microbiology 44, 149-156.

ENTIAN, K. D. (1977). Lack of carbon catabolite inactivation in a mutant of Saccharomyces cerevisiae with reduced hexokinase activity. Molecular and General Genetics 158, 201-210.

Fiechter, A., FuHRMANN, G. F. \& KÄPPELI, O. (1981). Regulation of glucose metabolism in growing yeast cells. Advances in Microbial Physiology 22, 123-183.

Geurts, Th. G. E., DE KoK, H. E. \& Roels, J. A. (1980). A quantitative description of the growth of Saccharomyces cerevisiae CBS 426 on a mixed substrate of glucose and ethanol. Biotechnology and Bioengineering 22, 2031-2043.

HaArasilta, S. \& Oura, E. (1975). On the activity regulation of anaplerotic and gluconeogenetic enzymes during the growth phase of baker's yeast. European Journal of Biochemistry 52, 1-7.

HERBERT, D. (1975). Stoicheiometric aspects of microbial growth. In Continuous Culture, vol. 6, pp. 1-30. Edited by A. C. R. Dean, D. C. Ellwood, C. G. T. Evans \& J. Melling. Chichester: Ellis Horwood. 
Holzer, H. (1976). Catabolite inactivation in yeast. Trends in Biochemical Sciences 1, 178-181.

KARRER, D. (1978). Der total gefüllte Bioreaktor. Ph.D. thesis, Eidgenössische Technische Hochschule, Zürich.

KNOEPFEL, H. P. (1972). Zum Crabtree Effekt bei Saccharomyces cerevisiae und Candida tropicalis. Ph.D. thesis, Eidgenössische Technische Hochschule, Zürich.

MEYeNBURG, H. K. VON (1969). Katabolit Repression und der Sprossungszyklus von Saccharomyces cerevisiae. Ph.D. thesis, Eidgenössische Technische Hochschule, Zürich.

Polakis, E. S. \& Bartley, W. (1965). Changes in enzyme activities of Saccharomyces cerevisiae during growth on different carbon sources. Biochemical Journal 97, 284-297.

Polakis, E. S., Bartley, W. \& MeEk, J. A. (1965). Changes in the activities of respiratory enzymes during the aerobic growth of yeast on different carbon sources. Biochemical Journal 97, 298-302.

SchatZManN, H. (1975). Anaerobes Wachstum von Saccharomyces cerevisiae. Ph.D. thesis, Eidgenössische Technische Hochschule, Zürich.

WitT, I., KronaU, R. \& HolzeR, H. (1966). Repression von Alkoholdehydrogenase, Malatdehydrogenase, Isocitratlyase und Malatsynthase in Hefe durch Glucose. Biochimica et biophysica acta 118, 522-537. 